\title{
LA GEOGRAFÍA MILITAR ESPAÑOLA, 1939-1945
}

\author{
POR
}

\author{
CLEMENTE HERRERO FABREGAT
}

Introducción

La geografía militar ha tenido una larga tradición en España desde principios del siglo XIX, estando caracterizada por estudios de alto nivel científico aplicados a la táctica y estrategia militares. Esta línea en la que sobresalen, entre otros, José Gómez de Arteche y Moro con su Geografía histórico-militar de España y Portugal, o Luis Villanueva López-Moreno, con un libro que constituye uno de los mejores estudios geográficos aplicados para la guerra, Bases para el estudio de la Geografía militar, tuvo su continuidad en la década de los cuarenta. En ese momento destaca la Geografía militar de España de Díaz de Villegas, que se escribió en 1936 y fue publicada en 1940; en ella, después de realizar un estudio geográfico general de España, pasa en la segunda parte a analizar su aplicación en los teatros de operaciones. Este mismo publicó en 1946 una obra de gran interés titulada La Geografía y la guerra. Estudio militar del terreno; en la misma examina todos los componentes del espacio geográfico físico, obteniendo conclusiones militares de cada uno de ellos. Puede considerarse a Díaz de Villegas el genuino representante de la geografía militar de la postguerra, aunque figuras como el general Aranda o el marino Luis Carrero Blanco se preocuparon por estos temas desde ópticas más estratégicas y políticas.

Clemente Herrero Fabregat. Escuela Universitaria de Formación del Profesorado. Universidad.Autónoma de Madrid.

Estudios Geográficos

Tomo LIX, n. ${ }^{\circ} 232$, julio-septiembre 
A esta continuidad de la Geografía militar hay que añadir que resulta importante conocer si los militares españoles durante el período 1939-1945 estuvieron influidos por las teorías geopolíticas alemanas en un momento de la vida política española en el que se dio una fuerte influencia de las potencias del Eje hasta el desembarco de los aliados en África del norte, noviembre de 1942, iniciándose un relativo distanciamiento con el pase de la no beligerancia a la neutralidad, previa destitución en Asuntos Exteriores de Ramón Serrano Súñer.

El objetivo de este artículo es conocer las características de la geografía militar de estos años caracterizada por una parte de la cúpula militar por reivindicaciones territoriales, reivindicaciones que fueron más intensas en los círculos políticos y periodísticos (Castiella y Areilza, 1941). En cambio en la mayor parte de la geografía netamente universitaria centrada en la revista Estudios Geográficos la penetración de las ideas geopolíticas expansionistas alemanas no tuvo prácticamente importancia tal como ha puesto de manifiesto Bosque (1992).

\section{Geografía militar española}

La geografía militar del período 1939-1945 se caracteriza por unos planteamientos de tipo técnico, algunas veces impregnados de referencias de la guerra civil, en los que los trabajos que podrían denominarse geopolíticos, mediante los cuales se realizaban demandas territoriales respecto a África que la propia dinámica de la Segunda Guerra Mundial arrinconó, tienen una relativa importancia cuantitativa. Los artículos dedicados a este tema, seleccionados de las revistas que más abajo se indican, suponen un 15,8 \% del total de la producción, el resto va encaminado a estudios geográficos y su aplicación estratégica y táctica, teniendo un alto porcentaje los dedicados al análisis de las operaciones militares referidas a la Segunda Guerra Mundial mediante unos estudios muy minuciosos.

El concepto de geografía militar aparece, como ocurría en la concepción geográfica anterior a la guerra civil, vinculado estrechamente a la geografía general por no ser ninguna ciencia independiente y distinta de la misma, sino «un mero punto de vista, una rama, una dirección, dentro de aquel orden total de conocimientos. A la Geografía General le preocupa el triple estudio del medio físico, biológico y humano. La Geo- 
grafía militar, basándose en ella, partiendo de sus afirmaciones, deduce su influencia en las operaciones de guerra y en las necesidades de los ejércitos en campaña». (Díaz de Villegas, 1940, p. 1).

En 1941 se señalaba respecto a la importancia de la geografía que «para el militar el conocimiento del terreno en que ha de operar es un arma más. El conocimiento de las líneas de comunicación, de la hidrografía, de la orografía, de los accidentes diversos, que el ejército ha de encontrar en su marcha, es absolutamente necesario que sea dominado por los jefes que han de ordenar las operaciones; por estas razones, es indispensable que el conocimiento geográfico sea base fundamental, al mismo tiempo que el histórico, de cualquier decisión de un mando para que ésta sea acertada» (Gazapo, 1941, p. 32).

El conocimiento del «terreno», término utilizado por los militares, plantea una cuestión ¿dicho conocimiento es necesario en los planteamientos estratégicos o en los tácticos? Muro Morales (1992), que ha estudiado el pensamiento militar sobre el territorio en la España Contemporánea, en el capitulo 15 de su obra traza con gran claridad la evolución del mismo desde finales del siglo XvIII a finales del Xx. Distingue previamente entre la estrategia militar o caudal de conocimiento previos, necesarios para desarrollar una práctica armada, y la táctica o ejecución de dicha práctica, siendo considerada la estrategia como la ciencia de la guerra, mientras que la táctica constituía el arte militar, de características eminentemente prácticas.

El máximo representante de la Geografía militar española del momento que se estudia, Díaz de Villegas, se sitúa dentro de la evolución del pensamiento militar sobre el territorio en la línea del prusiano Karl Clausewitz (1780-1831), decantándose por la táctica militar en la que se da una cierta valoración del territorio o terreno, afirmando que hay que divulgar los conocimientos generales de la Geografía militar, fuera incluso del Ejercito, debido a que la tierra pertenecerá a quien mejor la conozca. En este sentido hay que destacar que los estudios geográficos en Francia después de la guerra franco-prusiana adquirieron una gran importancia ya que se acusó a los soldados franceses de un total desconocimiento del terreno (Bullón, 1931, p. 17). Pero este reconocimiento de la importancia del terreno geográfico «no debe sin embargo, aparejar en nosotros un concepto fatalista, cual el de un determinismo exagerado fuera de todas proporciones (Díaz de Villegas, 1940, p. 2). 
Para Clausewitz, cuyo pensamiento en relación con los movimientos filosóficos ha sido analizado recientemente (Alonso Baquer et al. 1990) «la relación entre la guerra y el terreno es una de las más influyentes de entre las que se presentan en la conducción de una campaña militar, debido a que está presente constantemente, de modo que es imposible concebir que una acción bélica se produzca de otro modo que en un espacio definido, y es posible analizarla a diferentes escalas de observación ya que mientras por una parte puede alcanzar a los detalles más pequeños de la localidad, por la otra puede abarcar las más amplias extensiones del territorio» (Muro, 1990, p. 22). En esta línea un profesor de Geografía militar y Geología de la Escuela Superior de Guerra afirmaba en 1927 que se «acostumbraba a dividir los reconocimientos en tácticos o topográficos y estratégicos o geográficos, incurriendo en un manifiesto error, puesto que unos y otros, al estudiar el terreno caen bajo el dominio de la Geografía» (Villanueva, 1927, p. 173). De esta forma se puede establecer que la geografía es un elemento básico en el razonamiento militar tanto desde una perspectiva estratégica, que supone el planteamiento previo de la acción mediante mapas, como táctica, que lleva estos planteamientos a la practica en un terreno determinado.

El pensamiento geográfico militar español a través de la revista África y del Boletín de la Real Sociedad Geográfica (1939-1945)

La revista África, cuyo antecedente directo fue la Revista de Tropas Coloniales, empezó a publicarse en su tercera época en enero de 1942. Primeramente se subtituló Revista española de colonización, y desde el número 8, Revista de acción española, vinculándose a partir del número 16, abril de 1943, al Instituto de Estudios Políticos. El formato era de 33 por $23 \mathrm{~cm}$., y tuvo una portada permanente a lo largo de su existencia. $\mathrm{Su}$ periodicidad fue mensual durante el primer año, y posteriormente bimensual en algún número, normalmente en los meses de verano. Su redacción radicó en Madrid en la C/ Serrano, n. ${ }^{\circ} 16$, pasando posteriormente a Duque de Medinacelí, n. ${ }^{\circ} 4$, y desde febrero de 1945 a Juan de Mena, n. ${ }^{\circ} 15$. Los talleres de impresión fueron hasta junio de 1942 Afrodisio Aguado, desde esta fecha hasta febrero de 1945 Gráficas españolas, y posteriormente los talleres de la Delegación Nacional de F.E.T. y 
de las J.O.N.S. El precio de cada número era de 5 pesetas. El director de la misma fue el Teniente coronel José Díaz de Villegas, diplomado de Estado Mayor, que a veces utilizó el seudónimo de «Hispanus», hasta su nombramiento como Director General de Marruecos y Colonias en 1944, siendo sustituido por el Teniente coronel del ejército de tierra Gonzalo Gregori.

La revista tenía unas secciones permanente tituladas «Nuestra Atalaya y «Publicaciones» y en la contraportada unos mapas que solían representar los principales imperios coloniales, hechos histórico-geografícos, rutas geopolíticas, etc. A partir de 1943 apareció un suplemento titulado «África. Boletín de Información».

En ella colaboraron no solamente militares sino geógrafos, geólogos, e historiadores como Manuel Terán, Ramón Ezquerra, Eduardo Hernández Pacheco, Martín Almagro, Antonio Rumeu de Armas, entre otros, razón por la cual se presenta como una publicación con rigor científico, colaborando también personajes políticos como José María de Areilza, Fernando María Castiella, José Cordero Torres, etc. De la misma se han examinado 48 números, extrayéndose un total de 63 artículos escritos por militares, que hacen relación a temas geográficos y geográfico-militares.

El Boletín de la Real Sociedad Geográfica es una publicación de un gran valor para conocer la geografía española del momento ya que junto con Estudios Geográficos, revista propia del área universitaria, constituyen las dos publicaciones periódicas de la época. Reinició su publicación en 1941, con el tomo LXXXVII, cada tomo constaba de tres o seis números según fuese trimestral o semestral. Su formato es de 23 por $15 \mathrm{~cm}$. y ha mantenido su portada tradicional en verde con el título en negro de la misma. Su periodicidad fue trimestral durante 1941, pasando a semestral a partir de esa fecha. Su redacción radicaba en la sede de la Academia de la Historia, C/ León, n. ${ }^{\circ}$ 21, de Madrid Los talleres de impresión fueron Aguirre impresor. El precio de la suscripción era de 40 pesetas al año para la península, islas adyacentes, Marruecos y América y de 46 para la Guinea española y extranjero. El director de la misma era el presidente de la Sociedad, hasta 1943 el general diplomado de Estado Mayor Antonio Aranda Mata; en el tomo LXXIX aparece como presidente D. Pedro de Novo Fernández-Chicharro. Las secciones permanentes eran: Noticiario geográfico, Bibliografía, Revista de revista y Actas de sesiones. Se han analizado los tomos correspondientes al pe- 
ríodo 1941-1945 (t. LXXVII a t. LXXXI), extrayéndose 19 artículos escritos por militares sobre temas geográfico-militares.

En muchos artículos de estas dos revistas no se indicaba la pertenencia al ejercito de los autores o si estos eran Diplomados en Estado Mayor, por esta razón se han manejado el Anuario militar de España (1936), los Escalafoncillos de los cuerpos patentados de la armada (1940-1945), Cuerpos y servicios de Estado Mayor y auxiliares de Estado Mayor (1940-1945), y la Escalilla de las armas y cuerpos del ejército del aire (1940-1945). Se han utilizado los fondos de la Hemeroteca Nacional y los del Servicio Histórico Militar de Madrid.

De la época existe otra publicación de tipo mensual, la revista Ejército, subtitulada Revista ilustrada de las armas y servicios, cuyo primer número apareció en febrero de 1940. Se trata de una revista más divulgadora, sin numeración de las páginas en los primeros números, que por su carácter general no se ha incluido en este trabajo. No obstante, se publicaron algunos artículos sobre reivindicaciones territoriales o estudios militares sobre acontecimientos bélicos, guerra franco-prusiana, Primera Guerra Mundial, etc. Dicha revista ha sido estudiada desde la perspectiva de la ideología del ejército franquista en el período 1939-1959 por Losada (1990), y también es citada por Reguera (1991).

Para establecer una distribución temática de los artículos de geografía militar se han seguido los planteamientos de Díaz de Villegas, que la concibe, como hemos indicado, como un «punto de vista» de la Geografía general con unas conclusiones militares de cada una de sus ramas. Este planteamiento coincide, en sus líneas generales, con el que hizo Villanueva en uno de los mejores libros españoles de geografía militar escrito en 1927. En el capítulo II, apartado 13 crea un índice de ordenación geográfica, formando dos grandes apartados. El primero se dedica al estudio general del territorio en el que intervienen tres factores: físicos, humanos y militares. Dentro de los primeros establece las siguientes divisiones: posición geográfica; características geológicas, orográficas e hidrográficas; formas generales del suelo y su constitución; climatología, vegetación, aguas; producciones naturales. En los segundos destaca la situación histórica, población, comunicaciones, agricultura, industria, comercio; psicología, cultura, bellas artes; cuestiones políticas, sociales y económicas. En los terceros la potencialidad, utilización militar de las actividades y recursos; centros estratégicos, ob- 
jetivos; organización ofensiva y defensiva militar y naval; teatros de operaciones (naturales y circunstanciales); estudio militar de costas y fronteras. El segundo apartado se dedica a consideraciones militares sobre el conjunto del territorio y confirmaciones históricas (Villanueva, 1927, p. 32)

Mediante estos criterios se han agrupado los 82 artículos analizados, englobándose en dos grandes grupos. El primero comprende una serie de artículos que bajo el nombre estudios de Geografía general, local y regional facilitan informaciones geográficas de carácter básico: historia de la colonización española en África, etnogeografía, geografía agraria, actividades marinas y pesqueras, geografía de la salud, estudios locales y regionales, geografía histórica. Con todos estos conocimientos geográficos el militar tiene un bagaje de nociones que le permite entrar en los razonamientos propiamente relacionados con la guerra; por esta razón en el segundo grupo se incluyen los artículos de aplicación de los conocimientos geográficos a la actividad militar: climatología y economía aplicadas a la guerra, estrategia militar, terrestre, naval y aérea; esta estrategia se va a plasmar en lo que se denomina «teatro de operaciones» por lo que se agrupan una serie de artículos englobados bajo el epígrafe de operaciones militares, referidas básicamente a la Segunda Guerra Mundial. Por último existe un conjunto de artículos constituido por los que denominamos geopolíticos, en los que mediante argumentos geográficos se realizan peticiones territoriales (Cuadro I).

Dentro de los artículos de geografía general, local y regional hay 39, que suponen un $47,6 \%$ del total de la producción. Destacan los dedicados a estudios locales y regionales e historia de la colonización (13,4\% cada grupo) seguidos de los de geografía agraria y geografía histórica $(6,1 \%)$.

En los artículos englobados bajo el epígrafe aplicación militar (43), que suponen el 52,4 \% del total, destaca el grupo dedicado a Operaciones militares (24,4\%), seguido de los referentes geopolítica, tanto en su vertiente naval como terrrestre que suman 13 (15,8\%).

Se observa una tendencia en los artículos analizados a que un número muy pequeño de autores publiquen un elevado número de artículos. Este hecho puede acercar al concepto de productividad que se utiliza en bibliometría aplicado por Lotka en 1926, de acuerdo con el cual el número de autores que publican $n$ trabajos es inversamente proporcional 


\begin{tabular}{l|r|r|r|r}
\hline \multicolumn{5}{c}{ CUADRO I } \\
\multicolumn{4}{c}{ DISTRIBUCIÓN TEMÁTICA DE LOS ARTÍCULOS PUBLICADOS } \\
\multicolumn{3}{c}{ EN EL BOLETÍN DE LA REAL SOCIEDAD GEOGRÁFICA } \\
\multicolumn{1}{c}{ Tema } & África & B.R.S.G. & Total & Porcentaje \\
\hline \multicolumn{3}{c}{ Y REVISTA ÁFRICA } \\
\hline Geografía General & & & & \\
Historia colonización & 11 & - & 11 & 13,4 \\
Etnogeografía & 2 & - & 2 & 2,4 \\
Geografía de la salud & 3 & - & 3 & 3,6 \\
Geografía agraria & 5 & - & 5 & 6,1 \\
Pesca y marina & 1 & 1 & 2 & 2,4 \\
Estudios locales y regionales & 8 & 3 & 11 & 13,4 \\
Geografía histórica & 2 & 3 & 5 & 6,1 \\
Aplicación militar & & & & \\
Cartografía & & & & \\
Meteorología & 1 & 2 & 3 & 3,6 \\
Economía de guerra & - & 2 & 2 & 2,4 \\
Estrategia aérea & - & 1 & 1 & 1,2 \\
Evaluación territorial & 3 & - & 3 & 3,6 \\
Operaciones militares & 1 & - & 1 & 1,2 \\
Geopolítica naval & 19 & 1 & 20 & 24,4 \\
Geopolítica general & 2 & 4 & 6 & 7,3 \\
$\quad$ Total & 5 & 2 & 7 & 8,5 \\
\hline
\end{tabular}

a $n^{2}$, pero como el tiempo que se cubre es relativamente reducido, no es aplicable por lo que se enumeran únicamente los autores, con el número y porcentaje de artículos (Cuadro II).

La producción de los cuatro primeros autores supone un $43,8 \%$ total mientras que la de los autores con uno o dos artículos supone un 32,7\% del total de artículos. Otro aspecto que interesa destacar de los autores es si se encuentran en posesión del Diploma de Estado Mayor, del total de artículos escritos 52 corresponden a éstos, es decir un $63,4 \%$ del total, este porcentaje está distribuido prácticamente por igual en ambas revistas, un $65,1 \%$ en África y un 57,9\% en el Boletín de la Real Sociedad Geográfica. Es decir, que más de la mitad de artículos están escritos por militares con gran capacidad estratégica y táctica. 
CUADRO II

AUTORES MÁS PRODUCTIVOS

\begin{tabular}{l|c|c}
\hline \multicolumn{1}{c|}{ Autor } & N.' de art. & Porcentaje \\
\hline García Figueras, Vicente (E.M.) & 13 & 15,8 \\
Fontán y Lobé, Juan (E.M.) & 10 & 12,2 \\
Díaz de Villegas, José (E.M.) & 7 & 8,5 \\
Domenech Lafuente, Ángel & 6 & 7,3 \\
Carrero Blanco, Luis (E.M.) & 5 & 6,1 \\
López de Castro, Celestino & 4 & 4,9 \\
Munaiz Brea, Ricardo (E.M.) & 4 & 4,9 \\
Aranda Mata, Antonio (E. M.) & 3 & 3,7 \\
Flores Morales, Ángel & 3 & 3,7 \\
Bullón Díaz, Galo & 2 & 2,4 \\
Dicenta Seguí, José & 2 & 2,4 \\
Gregori Peiró, Gonzalo & 2 & 2,4 \\
Lombardero, Manuel & 2 & 2,4 \\
Mateo Marcos, Santiago & 2 & 2,4 \\
Rotaeche, Jesús María & 2 & 2,4 \\
15 autores con un artículo & 15 & 18,3 \\
\multicolumn{1}{c}{ Total artículos } & 82 & \\
\hline
\end{tabular}

La geografía general, local y regional como fuente de información para la geografía militar

Los artículos incluidos en este grupo tratan de trabajos típicamente geográficos sin grandes aplicaciones militares. No obstante, destaca que en gran parte de ellos no se sigue el esquema universitario del análisis geográfico, a pesar de lo cual se realizan estudios importantes que podrían calificarse de geografía aplicada. Se observa en algunos una relativa influencia del ambientalismo determinista aunque no se menciona a Ratzel.

En un primer grupo incluimos 11 artículos que se dedican a estudiar la historia de la colonización de los territorios españoles en África, 6 se centran en Guinea, 2 en el Sáhara español, y 1 en Sidi Ifni, todos ellos publicados en la revista África. Sobre el protectorado marroquí no se escribió ningún artículo ${ }^{1}$. De estos artículos, 7 se escribieron después del

${ }^{1}$ Únicamente se escribió un artículo sobre Marruecos español por el coronel Celestino M. López de Castro que se ha incluido, por su temática, en el apartado operaciones militares. 
desembarco aliado en África del Norte, posiblemente para afirmar la «españolidad» de estos territorios. Se trata de trabajos que están muy bien documentados tanto a nivel cartográfico como fotográfico, reproduciéndose, además, textos de las épocas en que estos territorios fueron colonizados por España; no obstante, a pesar del aspecto propagandístico que en ellos subyace, se da un planteamiento científico correcto. También en la revista Ejército, como ha señalado Losada, los artículos en los que se reclamaban nuevos territorios dejan paso a otros en los que únicamente se pide que España permanezca donde está, «glosándose la enorme labor desarrollada por España en sus colonias, los progresos aportados en su población, los recursos elevadores del nivel de vida que España proporciona, etc.» (Losada, 1990, p. 185). Todos estos artículos no pueden considerarse típicos de la geografía colonial, en la que la labor del geógrafo era previa al proceso de colonización, tal como se indicó en I Congreso Español de Geografía Colonial y Mercantil de 1883 (Rodríguez, 1996, p. 85).

A este conjunto de artículos sobre el proceso de colonización, temáticamente le siguen dos dedicados a estudios raciales, que entrarían de lleno en una rama geográfica que en los primeros años del siglo estaba de moda, la etnogeografía. Un artículo se titula «El tipo humano en nuestro territorio de Ifni» (Linares, 1943), y otro «Las poblaciones negras del África ecuatorial» (Fontán, 1942a). Del primero es autor un capitán médico del ejército, y del segundo el Director de Marruecos y Colonias. Van acompañados de gráficos, planos de viviendas y poblados, fotografías, dibujos anatómicos, etc. En ellos se analiza el origen de la población, las características psicogeográficas, dándose una influencia ratzeliana. La psicogeografía se consideraba a principio de siglo como una «parte de la Antropogeografía, en la que se estudian, bajo su aspecto geográfico, las diferenciaciones de orden intelectual y moral entre los hombres» (Moreno, 1912, p. 174). Se establece una diferencia entre los beréberes Ait Ba-Amram, habitantes de Sidi Ifni, y los negros de Guinea. Sobre los primeros aplicando los criterios raciales de la «ciencia germana» se destaca su pureza racial, por no haberse mezclado con otros grupos étnicos, valorando su «generosa aportación personal a nuestra gloriosa Cruzada» (Linares, 1943, p. 36). Al analizar las características de la población negra, se adopta una perspectiva ambientalista afirmándose que el estudio del indígena no puede hacerse fuera de su medio por existir reacciones mutuas entre el medio y el hombre, características que si 
no se tienen presentes falsearían cualquier análisis. De la población negra se destaca su estoicismo ante las enfermedades, la adaptación de la vista y del oído al medio que nunca es superior a la del europeo, su sentido de equidad, su indiferencia ante el progreso, que no entiende desde su propio ambiente, su mínimo dominio del pensamiento abstracto, y, sobre todo, se realiza una curiosa interpretación de la antropofagia que practica (Fontán, 1942a, p. 3).

Se completa este análisis etnogeográfico con tres artículos que estudian la influencia del medio en la salud, agrupados bajo el epígrafe de geografía de la salud, en ellos se analiza la labor colonial realizada por España en Guinea, destacando dos artículos que se centran en el parasitismo intestinal provocado por el anquilostoma y en el paludismo, enfermedades endémicas de Guinea, dándose una serie de soluciones médicas (Dicenta, 1945 y 1945a).

Otro grupo de 5 artículos, publicados en la revista África, está dedicado a estudios agrarios. Son trabajos que no siguen el esquema clásico de los estudios universitarios por considerar los problemas agrarios y de abastecimiento de agua en función de unas soluciones concretas; por esta razón podrían calificarse de trabajos propios de geografía aplicada. Se estudian las condiciones naturales de las principales producciones de Sidi Ifni y Guinea, dándose alternativas técnicas a los distintos problemas planteados por el medio. Por ejemplo respecto a la extensión del regadío en Sidi Ifni, se afirma que en su resolución deben participar «el meteorólogo, evaluando y calculando las probabilidades de lo que dará la atmósfera; el geólogo, buscando la riqueza del subsuelo; el ingeniero, estudiando y realizando las soluciones técnicas, el botánico y el agrónomo buscando la mejor utilización del agua disponible; el jurista, estudiando las condiciones de las tierras; el administrador, previendo el problema social porque el número de litros-segundo determinará la cadencia del mejoramiento en la vida, y los limites del crecimiento de la población en el territorio» (Domenech, 1945, pp.9-10).

Esta misma característica de geografía aplicada se encuentra en un grupo de dos artículos, correspondientes a cada una de las dos revistas estudiadas, sobre actividades marinas y pesqueras, uno sobre la marina mercante y su importancia en la economía mundial (Rotaeche, 1942) y otros sobre la pesca en Sidi Ifni. Este último realiza un pormenorizado análisis de las características geográficas de la pesca que practican los Ait Ba Amram, en el que destaca la pobreza del territorio en relación 
con el suelo y el régimen de lluvias, que lleva a los habitantes de este territorio a practicar la pesca, en unas zonas relativamente templadas por la existencia de la corriente fría de Canarias. El problema radica en la existencia de borrascas atlánticas que provocan fuertes marejadas centradas en los meses de septiembre a mayo. Se estudian con gran detalle los tipos de embarcaciones, los aparejos de la pesca, los productos obtenidos, acompañando una serie de fotografías, planos, y diferentes mapas temáticos, distinguiéndose uno sobre el consumo del pescado en el sudoeste marroquí (Domenech, 1944).

Otro grupo de 11 artículos está dedicado a realizar estudios monográficos regionales, 2 sobre Ifni y Sahara y 3 sobre la isla de Madagascar. No se trata de estudios de evaluación de la potencialidad de un país desde la óptica militar sino de descripciones que siguen el esquema propio de la geografía regional. A estos estudios se unen 6 trabajos de tipo local, 4 sobre Marruecos, y 2 sobre Sevilla.

Por último, hay 5 trabajos de geografía histórica entre los que destaca uno del general Aranda (1942) titulado «Geografía cidiana» en el que se muestra partidario de un cierto determinismo geográfico, esta postura determinista la mantendrá en otro artículo suyo que analizaremos en el apartado dedicado a la geopolítica.

\section{La cartografía, elemento básico de la estrategia y táctica militar}

En este grupo se incluyen los artículos que hacen referencia a la cartografía militar. El mapa, tal como puso de manifiesto hace más de dos décadas Yves Lacoste (1977), es un instrumento de dominación del espacio, debiendo cada hecho ser analizado y representado según la magnitud del hecho que se representa, que se convierte en un espacio de conceptualización geográfica.

En 1941 Darío Gazapo, Coronel de Estado Mayor, señalaba que «la carta militar, como fundamento indispensable para asegurar la vida y la defensa de la nación, ha de tener, naturalmente, características especiales, zonas reservadas, formas y detalles precisos para los mandos militares que no interesan al elemento civil», estas cartas debían estar realizadas a diferentes escalas, siendo la fundamental la correspondiente a 1:50.000 ya que de ésta «sacaremos después el 1:100.000, que es el mapa del Mando, y después el 1: 200.000 y 1:400.000, que son hojas que

$$
-454-
$$


conviene desde el punto de vista estratégico y de comunicaciones. Además hemos de propugnar porque en ese plano en publicación a escala 1: 50.000 se disponga de las minutas de campo a escala 1: 25.000, para que sirvan de base al elemento artillero, porque en ellos fundamentan su teoría y práctica de tiro. Y luego, por último, ya se tratará de las escalas inferiores, por ejemplo, de 1:10.000, que serán planos que convengan a ciertos accidentes geográficos; el 1: 5.000 para campamentos y campos de tiro, etc. (Gazapo, 1941, pp. 41-42).

Destacan tres artículos, uno de tipo general, el citado del coronel Gazapo, y otros dos del Coronel de Estado Mayor Manuel Lombardo Vicente $(1944,1945)$, jefe del Servicio Geográfico del Ejército, sobre los trabajos cartográficos realizados en África española.

El valor estratégico y táctico de la cartografía en el período que se analiza se ve demostrado en el acta de sesiones celebrada el 8 de febrero de 1943 de la Real Sociedad Geográfica de Madrid, en dicha acta se recoge textualmente una comunicación recibida con fecha 4 de diciembre de 1942, en la que la Dirección General de Bellas Artes transmite otra del Ministerio del Ejército en que «se comunica el carácter reservado que tiene el mapa del Sahara Español y regiones inmediatas a escala 1:1.000.000 formado por D. Enrique D'Almonte y publicado por la Real Sociedad Geográfica en 1914, por lo que esta entidad deberá reservar o entregar al Archivo del Servicio Geográfico del Ejército los ejemplares que del mismo posea» (Torroja, p. 279). La aportación castrense a la cartografía española ha sido estudiada por Alonso Baquer (1972).

\section{Aplicación militar de la meteorología}

Un grupo de 27 artículos, excluidos los cartográficos, engloba los trabajos dedicados a analizar la aplicación militar de los conocimientos geográficos. En este sentido se considera la geografía militar como una ciencia aplicada, que fue definida como «la ciencia que estudia la influencia y utilización de los factores geográficos en la guerra» (Villanueva, 1927, p. 27).

Los artículos dedicados al factor meteorológico inciden en la importancia que tiene éste no solamente en la aviación sino en la artillería, estudiándose las principales masas de aire establecidas por Bjerknes. El estudio de la meteorología debe atender «a la física de la atmósfera, la 
termodinámica, la mecánica de los fluidos, el cálculo de probabilidades, los sondeos de la alta atmósfera, la unidad de criterio en las observaciones, el aumento de densidad de los puestos de observación para hacer progresar el valor matemático de las informaciones meteorológicas. Esta visión permite establecer los objetivos militares de la meteorología, que son: las observaciones de tiempo pasado, las condiciones atmosféricas reinantes, la previsión de tiempo a largo y corto plazo deducida del diagnostico de la situación actual, y las investigaciones espaciales, generalmente de orden micrometeorológico» (Carrero, 1941, p. 56). Por esta razón se afirmaba que «el conocimiento del factor meteorológico es tan indispensable, que el servicio encargado de asegurarlo debe formar parte de todo Estado Mayor» (Nuñez, 1942, p. 349).

En relación directa con la meteorología se analizan las rutas aéreas africanas y su interés estratégico en tres artículos del Teniente coronel de aviación, diplomado en Estado Mayor, Ricardo Munaiz. Estudia primeramente la penetración aérea en el continente africano a nivel militar, deportivo y comercial, destacando la presencia francesa en función de su importante imperio colonial al afirmar "que parece inútil subrayar la importancia que tiene este imponente esqueleto de la red aérea francesa en África, en orden a la penetración pacífica del territorio, al desarrollo económico de los dominios, a su rápido enlace entre sí y con la metrópoli y, en caso de guerra, al apoyo de operaciones terrestres y transportes de fuerzas aéreas».(Munaiz, 1942a, p. 19). La teoría del espacio vital es aplicada a África al analizar en un artículo titulado «El aire africano y su interés estratégico» (1942). Estudia la situación de las naciones democráticas, a las que denomina naciones unidas, de la Francia de Vichy y de las naciones del Eje. Respecto a la potencia aérea defensiva y ofensiva española propone sobre un mapa del Viejo Continente un estudio estratégico para conocer el radio de acción de la aviación (Munaiz, 1942, p. 21).

\section{Evaluación militar de territorios geográficos.}

Teatros de operaciones militares

Sobre trabajos globales de evaluación de un posible teatro de la guerra destaca un artículo sobre las condiciones geográficas del desierto, analizándose los problemas que para el militar este medio presenta y las 
condiciones exigidas a las tropas europeas, y las soluciones que el Estado Mayor debe dar tales como «la cuestión del abastecimiento del agua y forraje, así como la del utensilio, vehículos, monturas y equipos de animales» (Paschasius, 1942, p. 35), sin los cuales no se puede emprender una marcha; observándose como la geografía militar tiene este carácter de aplicación de los conocimientos geográficos.

En los artículos sobre operaciones militares destaca el teniente coronel Vicente García Figueras, diplomado en Estado mayor, que escribió 13 sobre «el salto de África a Europa» en la revista África desde septiembre de 1943 hasta noviembre-diciembre de 1944. El tema de la Segunda Guerra Mundial, por la importancia que tenía para la política española, es tratado con gran detalle y profundidad, evolucionando el tratamiento del mismo al ritmo de la evolución de los hechos bélicos. Así, por ejemplo, Carrero Blanco escribió en 1945 un artículo que puede calificarse de aséptico sobre el desarrollo de la guerra en África, en el que no muestra la admiración anterior por el Eje. Por esta razón, puede afirmarse que ciertos aspectos de la geografía militar mas relacionados con aspectos políticos presentan un carácter coyuntural en el que los acontecimientos políticos marcan su ritmo, frente a los análisis propiamente estratégicos en los que los planteamientos conceptuales y metodológicos son más permanentes. En esta línea hay que situar un conjunto de artículos en los que se realizan reivindicaciones territoriales africanas mediante argumentaciones geográficos, además de realizar una gran valoración del mar como centro de poder.

\section{Importancia geopolítica del mar}

El conocimiento de las condiciones marítimas es junto con la meteorología un factor importante dentro de la estrategia naval. Este grupo está compuesto por 6 artículos, si se incluye uno dedicado a la oceanografía en el norte de África. Destaca como autor de 3 artículos el entonces capitán de fragata Carrero Blanco, que se encuentra en la línea del historiador y marino A. T. Mahan que en su libro The influence of the Sea Power upon the French revolution and Empire, publicado en 1882, recalcó la importancia que el dominio de los mares había tenido y tenía en la historia de la humanidad. Carrero establece una relación directa entre la geografía y la actividad naval al afirmar que «tan íntima es 
la relación entre la Geografía y la actividad bélica en el mar, tan abrumadoras son las servidumbres de tipo geográfico que pesan sobre cuanto a la guerra naval se refiere, que bien pudiera decirse, sin temor a incurrir en gran exageración, que la estrategia naval es geografía pura» (Carrero, 1941a, p. 235).

A partir de esta afirmación, y después de un recorrido histórico en el que destaca la importancia del mar en la historia de la humanidad, establece dos situaciones una en tiempo de paz y otra en guerra. Respeto a la primera afirma, no exento de un cierto organicismo, que los centros productores con los industriales y de consumo constituyen una «especie de sistema arterial semejante, y tan vital, al que sirve para la circulación de la sangre en el cuerpo humano» (Carrero, 1941a, p. 240). Pero cuando los recursos de la diplomacia fallan y se entra en un proceso bélico, la flota de guerra tiene como misión asegurar la libertad de las comunicaciones marítimas propias e impedir que el enemigo disfrute de ellas, junto con la seguridad que debe dar al litoral, cooperando tanto en el orden estratégico como en el táctico con el ejército de tierra. $\mathrm{Al}$ anular a la flota adversaria se obtendrá el dominio del mar, «una vez logrado este, la explotación de este dominio se traduce inmediatamente en disponer libremente del tráfico comercial a través del mar, en tener asegurado el litoral propio, en poder cooperar en todos los aspectos con el ejército de tierra y en impedir que el enemigo pueda conseguir nada de esto» (Carrero, 1941a, p. 237). En función con esto establece las características de una flota moderna y su valoración en razón de la situación geográfica.

En relación con la importancia que se da al dominio marítimo destaca la situación geopolítica de España, a caballo entre dos mares vitales y dos continentes, y la necesidad de dotarse de una flota militar que sirva de unión de todos los territorios extrapeninsulares, añadiendo además una idea que suele estar presente en algunos razonamientos militares, que «España es un península que se acerca mucho más a una isla en orden a su amplitud y posibilidades de intercambio de su unión con el continente europeo, dado que la casi totalidad de los 677 kilómetros de frontera con Francia corresponden a la abrupta orografía de la cordillera pirenaica teniendo únicamente tres líneas de ferrocarriles, a todas luces insuficientes para abastecer a la nación. Por estas razones afirmando en el más puro estilo militarista que «el ser o no ser de España que depende del ser o no ser de su potencia militar, está condicionado a la existencia 
o no de su efectivo poder naval» (Carrero, 1943, p. 101). Por ello es necesaria una flota mercante capaz de abastecerla y una flota militar que asegure a la primera su libertad de acción. En este sentido se observa una influencia de las ideas regeneracionistas recogidas en el mencionado Congreso de Geografía Colonial, en el que fue aceptado por todos los participantes la necesidad de proceder a potenciar la marina mercante por todos los medios disponibles, ya que de ella dependería el proceso de expansión del país (Rodríguez, 1996, p. 85). Las tesis de Carrero fueron llevadas a la practica por el Programa naval, 1943, puesto en marcha por J. A. Suanzes (Reguera, 1991, p. 42).

Por tanto, para él el dominio del mar es fundamental, aunque respecto a la Segunda Guerra Mundial y debido a la situación de amistad de España con Alemania realiza una extraña argumentación que le lleva a afirmar «que la situación general de Alemania es distinta, no está cercada como en la Gran Guerra y cuenta con los recursos del continente. El Pacto de no agresión con Rusia melló, al comenzar la lucha, el arma de Inglaterra, que representa el dominio del mar». No obstante, «Europa está bloqueada por mar. Para vivir sin el mar, los ejércitos del Reich se han apoderado de todos los centros productores e industriales del continente europeo». Por estas razones «las potencias europeas del Eje luchan pretendiendo prescindir de los Océanos. Sus objetivos son: acabar con Rusia disponiendo de todas las riquezas continentales, y cerrar Suez para soldar, a través del mediterráneo, África del Norte a la economía europea» (Carrero, 1942, pp. 3-4). Por todo ello concluye «que si dependiera de las comunicaciones marítimas perdería la guerra como la perdió en 1918 a pesar de la potencia de su ejército (Carrero, 1941a, pp. 257-258).

Dentro de la importancia que en los estudios estratégicos se da al dominio del mar, destaca un artículo de Díaz de Villegas en el que se analiza la importancia de los estrechos marítimos que es «económica, política, marítima y militar. Es económica, porque los estrechos relacionan siempre las tierras fronteras y abren así una vida natural al tráfico continental, terrestre y aéreo. Es marítima, porque son a su vez encrucijadas por donde desfila, densamente, el tráfico mundial, constituyendo puntos focales de la máxima importancia, es decir, lugares en donde el tráfico marítimo se concentra. Es militar, porque sólo la posesión de amplias tierras fronteras garantiza la seguridad de las tierras propias. Y es política, porque la posesión de los estrechos en toda su extensión es un 
instrumento magnífico que revaloriza la política del Estado» (Díaz de Villegas 1942, pp. 231-232).

Por estas razones estudia el valor geopolítico de los grandes estrechos de la Tierra, analizando los mismos en función de la guerra mundial. Recogiendo lo que Reynaud llama «Ecuador de contracción», un gran círculo de fracturas que envuelve el mundo, sitúa la circulación que se da siguiendo los paralelos en la que el canal de Panamá, el estrecho de Gibraltar, el canal de Suez, Adén y Singapur son puntos neurálgicos, estando todos ellos, salvo Panamá, bajo la vigilancia británica, indicando mediante unos gráficos geopolíticos las mercancías que circulan por los mismos. A parte de esta línea neurálgica de comunicación estudia los estrechos bálticos y las razones geopolíticas de la ocupación de Noruega y Dinamarca por parte de Alemania; la importancia del canal de la Mancha, y la tradicional presión de Rusia sobre el Bósforo, mar de Mármara y Dardanelos para buscar una salida al mar Mediterráneo, pretensión que siempre ha encontrado la más cerrada oposición de Inglaterra, aspecto que analizaría en 1950 Vicens Vives en su Tratado de Geopolítica.

\section{Geopolítica expansionista militar}

Como se ha indicado hasta ahora, la geografía militar española en el período 1939-1945 se caracteriza por estudios geográficos de tipo técnico, tanto tácticos como estratégicos, siguiendosee la línea de las geografía militares del regeneracionismo «en las que no se produjo una influencia clara de las doctrinas geopolíticas, entonces en boga en las grandes potencias» (Alonso Baquer, 1977, p. 277). No obstante, un sector de su cúpula militar durante el período 1939-1942 realizó una serie de planteamientos típicamente geopolíticos. Porcentualmente los artículos que plantean demandas territoriales representan el 8,5\% del total de la producción analizada. Pero este porcentaje hay que matizarlo ya que los 7 artículos incluidos bajo este epígrafe corresponden a militares de alta graduación relacionados directamente con la geografía, José Díaz de Villegas y Antonio Aranda Mata ambos diplomados en Estado Mayor, y al Director General de Marruecos y Colonias, el capitán de fragata diplomado en Estado Mayor, Juan Fontán y Lobé; se dió, por tanto una difusión de la geopolítica alemana, que dejó de practicarse a partir de 1943, y, sobre todo, a partir del final de la Segunda Guerra Mundial.

$$
-460-
$$


La afirmación de Díaz de Villegas de que la geopolítica «no se escribe en los libros: se hace a cañonazos sobre todo en el ámbito del mundo. $Y$ es que la guerra es, a la postre, el tribunal supremo ante el que fallan, en última instancia, los litigios y los pleitos de los pueblos», muestra dicha penetración. El planteamiento biologicista que hace dicho geógrafo militar revela una influencia coyuntural, que en su obra anterior y posterior está mucho más matizada, de las teorías geopolíticas alemanas. Escribe textualmente» El escolasticismo científico debate entre si la geopolítica pueda ser, como dicen unos, la ciencia del nacimiento, del desarrollo y de la muerte de los estados, o, como quieren otros, la ciencia de la sangre (la raza) y del suelo, o, como se dice también la ciencia que relaciona el estado social con el espacio geográfico. A la postre, todo viene a ser uno y lo mismo. Lo que importa señalar es el hecho biológico de cómo la célula social, al crecer, produce, con las demás que constituyen el tejido histológico del mundo, zonas de tensión y de fricción, que unas veces se manifiestan en estado larvado y otras termina engendrando choques económicos y militares», afirmando más adelante que «la geopolítica es la ciencia del estadista. La estrategia, la del caudillo militar. Pero ni éste ni aquel pueden actuar con independencia» (Díaz de Villegas, 1942, pp. 203-204). Recoge, así mismo, conceptos típicamente geopolíticos como el valor de la montaña y del llano, al afirmar «que la geopolítica nos habla de la influencia disgregadora de la montaña y, en cambio, del papel unificador del llano. Cierto Capitán ha dicho que en todo gran país llano radica el germen de un imperio. Pero la función unificadora del llano se hace patente por los ríos. El mismo papel, en efecto, que en lo físico realizan los ríos llevando al cauce las aguas que lateralmente reciben de las vertientes lo realizan desde un punto de vista geopolítico y de relación humana» (Díaz de Villegas, 1942, p. 205).

En los artículos analizados se reivindica un «espacio vital» español en el Norte de África, si bien no con la rotundidad y extensión de las peticiones de otros políticos y periodistas de la época. Estas reivindicaciones están en relación directa con la situación de España con respecto a las grandes corrientes del tráfico mundial; pero, no obstante, esta situación es contrarrestada por obstáculos de índole política ya apuntados por Díaz de Villegas en su Geografía militar de España. Dichos obstáculos son «tres cuartas partes del litoral atlántico aparecen ocupadas por la faja portuguesa, y en Portugal está el mejor puerto atlántico 
peninsular: Lisboa. La presencia inglesa en Gibraltar, hace de esta pequeña posesión inglesa el polo de atracción del tráfico internacional del Estrecho, convirtiendo a la plaza en el principal puerto de escala del Mediodía peninsular. La expansión francesa norteafricana, al sur de la zona costera de nuestro Protectorado, contrarresta, dentro de ciertos límites, al buscar salida al mar libre por Argelia y Casablanca, la función que parece haber reservado la Geografía a nuestro país, haciendo de él un puente continental del intercambio africano-europeo» (Díaz de Villegas, 1940, p. 22).

Las reivindicaciones que se realizan se apoyan históricamente en el reparto de África, en el que España prácticamente no estuvo presente, incidiéndose en el poco interés mostrado en las naciones europeas por el continente africano, frente a la labor evangelizadora y espiritual española. Fontán y Lobé, capitán de fragata y diplomado de estado Mayor, analiza la superficie, población y las producciones por habitante de los países colonizadores, indicando que «conjugando los factores espirituales y materiales, morales y económicos, se podrá asignar, en plena justicia, a cada nación el espacio vital que necesite para tener asegurada su existencia y se acabará para siempre con la injusticia que supone que haya naciones a quienes les sobra todo, mientras a otras les falta hasta lo más inmediato». Por esta razón espera que al igual que se ha instalado un nuevo orden en Europa, se inicie con rapidez un nuevo orden en África, preguntándose «¿Cómo se hará el nuevo reparto? No podemos predecirlo; pero si podemos asegurar que debe fundarse en la justicia ¡Que no se ha derramado tanta sangre en Europa, desde 1936, en que empezó nuestra Cruzada, para llegar a un orden nuevo injusto como el anterior!» (Fontán, 1942, p. 5)

Todo el problema hay que encontrarlo, según Díaz de Villegas, en una serie de tratados y conferencias (1902, 1904, 1906 y 1912) en los que Francia e Inglaterra relegaron a España, afirmando este geógrafo militar que «nuestra tesis es bien clara. España quiere un Marruecos sólo. Un Mogreb indivisible. Ningún reparto podría justificar la geografía o la historia. Ninguna división podría justificarnos la política parisina. España, como siempre, mantiene una tesis. La de la unidad marroquí. No hay más Marruecos que uno. Y ese Marruecos, por tanta y tanta razón, debe ser tutelado por España sin proindivisos» (Díaz de Villegas, 1942a, p. 7).

Las razones que se esgrimen para la fundación de este Imperio hispano-africano son de dos tipos: Marruecos constituye la continuidad de 
España, y las desproporciones existentes dentro del Mogreb exigen la aplicación de la teoría del «espacio vital». Dentro de las primeras en la conferencia inaugural del curso 1941-42 de la Real Sociedad Geográfica, publicada tanto en el Boletín de la misma como en África, el general Aranda, que en 1944 pasaría a disponible por su enfrentamiento con el general Franco, afirmaba que existía una unidad geológica, geográfica, racial, productora y cultural entre España y Marruecos, y en el más rancio determinismo decía «Nadie discute la unidad geológica del Sur de España y el Norte de África, y como la Geología es la madre de la Geografía, y ésta a su vez, engendra la Historia, basta recorrer las montañas y los valles marroquíes para comprender que no hay otro pueblo en el mundo, y especialmente en Europa, tan indicado como el nuestro para cumplir en Marruecos la misión protectora, y tan naturalmente apto y preparado para realizar una vez más la fusión espiritual y material que sólo bienes puede reportar a ambas naciones». Se plantea Aranda que pueden existir diferencias, que «no se oponen en nada a la idea fundamental de unidad de destino» (Aranda, 1942a, p. 22). Esta unidad se basa en una unidad racial dentro del propio Marruecos, predominancia de los beréberes puros y arabizados y mínima influencia hebrea y europea. Llegando a afirmar «por lo que respecta a la población de España, su continuidad de origen con las masas beréberes y la continua fusión a que ha estado sometida durante siete siglos son seguramente más de lo necesario para asegurar un grado de aproximación rayano en muchos casos en la igualdad completa. El marroquí siente profundos lazos de sangre que le unen a sus compatriotas..., conociendo de manera unánime, el parentesco próximo del español» (Aranda, 1942, p. 25). Se aplica de esta forma la teoría haushoferiana de la geopolítica como ciencia de la sangre y el suelo.

En el mismo sentido se expresaba Díaz de Villegas, quien aplicando el esquema de los países alpinos indica como los Montes Béticos y el Atlas constituyen una única unidad con sus dos prefosas el valle del Guadalquivir y el valle del río Sebú. Pero a esta estructura geomorfológica añade otra histórica, analizando las relaciones históricas desde la prehistoria entre el norte de África y la cultura almeriense, que se continuaron a lo largo de la historia pero que encontraron dos «obstáculos extraños en el cumplimiento de los que hemos visto era simplemente una misión histórica y un dictado natural. Estos obstáculos son Inglaterra, desde Tánger a Gibraltar, y Francia, entrometida primero en la expedi- 
ción de Argel, en Túnez, y en Marruecos más tarde» (Díaz de Villegas, 1942b, p. 9).

Junto con esta visión geográfica impregnada del más puro determinismo, Aranda realizó en el artículo mencionado un razonamiento geopolítico basado en la teoría del espacio vital. Compara la producción entre el Marruecos francés y español mediante mapas y estadísticas, observando un fuerte desequilibrio entre ambos, a favor de Francia. En función de dicho desequilibrio reivindica un espacio vital para los beréberes del Protectorado afirmando «que sería muy difícil hallar otro caso más acusado de urgente e indispensable aplicación de las teorías de la Geopolítica ya que los 720.000 habitantes de la zona española del Protectorado no disponen de posibilidad alguna de aumento ni elevación sensible de su nivel de vida (bien bajo), ni la zona tiene posibilidades de transformación adecuada, con una densidad de población triple que en la zona francesa, y que ya antes de la implantación del Protectorado obligaba a emigrar temporalmente a una buena parte de la población del Rif, a fin de procurar el sustento para el invierno» (Aranda, 1942a, p. 28).

Todas estas demandas desaparecieron prácticamente en las revistas analizadas, como se ha indicado anteriormente, a partir de 1943. Por tanto, puede afirmarse en función del material estudiado, que si bien hubo una influencia de las teorías geopolíticas nacionalsocialistas en un sector de la cúpula del ejército, estas se disiparon en el transcurso de la guerra mundial, y tuvieron una carácter coyuntural, siguiéndose la trayectoria técnica que caracterizó tradicionalmente el pensamiento geográfico de las fuerzas armadas. Por ejemplo, en 1944 Díaz de Villegas publicó un artículo en la Boletín de la Real Sociedad Geográfica, fruto de una conferencia impartida el 30 de octubre de 1944, titulado «España en África» que realiza un perfecto estudio geográfico con 19 gráficas y mapas temáticos, analizando las aportaciones españolas en el conocimiento geográfico del continente, estando las reivindicaciones territoriales muy matizadas ya que únicamente se compara el territorio y la población que España posee en África con el resto de la potencias coloniales, haciéndose referencia a los tratados de 1903 y 1912 con la merma territorial que este último supuso. El mismo Díaz de Villegas en su Geografía militar de España, escrita en 1936 pero publicada en 1940, al hablar de la raza hispana, sustento de la teoría sangre-suelo, decía «es, por tanto, una raza sintética la nuestra, sometida a influencias tan heterogéneas que Haunter Chamberlain ha podido decir que la raza española es una raza de

$$
-464-
$$


caos» (Díaz de Villegas, 1940, p. 58). También, para afirmar el carácter coyuntural de la geopolítica expansionista de algunos militares, se puede recoger una conclusión del mismo geógrafo militar respecto a Marruecos en la que la tan traída unión racial, geográfica e histórica con España no aparece al afirmar que «Marruecos resulta ser un todo definido, independizado por el Atlas y el Sahara del resto de África, mientras que sus fronteras marítimas y la cadena rifeña aíslan al país del mediterráneo, y las barras del litoral atlántico han constituido hasta la fecha un obstáculo serio a la navegación oceánica. Todo ello explica el hermetismo marroquí y la incomunicación de este país con el resto del mundo hasta mediados del siglo último» (Díaz de Villegas, 1940, pp. 403-404).

Carrero Blanco en un artículo publicado en 1945 «Síntesis del desarrollo de la guerra en África» realiza un estudio desde su óptica de marino, que puede clasificarse de objetivo sin muestras de grandes simpatías hacia las potencias del Eje. Para él dichas potencias cometieron durante la contienda una serie de errores tales como no invadir Inglaterra, y sobre todo no soldar Europa al continente negro mediante la plena posesión del mediterráneo oriental y «asegurar sus dos puertas: canal de Sicilia y Aden o al menos Suez esto requería ocupar las posiciones geográficas en que las fuerzas navales y aéreas de la Gran Bretaña podían apoyarse» (Carrero, 1945, p. 487).

Frente a esta geopolítica militar de carácter coyuntural, se dio una geopolítica científica, con fuertes influencias alemanas, cuyo máximo representante fue el profesor Vicens Vives (1940), y otra de tipo propagandístico desarrollada en ambientes políticos, universitarios y periodísticos de la época, que merece un estudio aparte.

\section{BIBLIOGRAFÍA}

AlONSO BAQUer, Miguel (1972): Aportación militar a la Cartografía española en la Historia Contemporánea, Madrid, Patronato Alonso de Herrera, Instituto de Geografía Aplicada.

- (1977): «La geografía militar en la hora del regeneracionismo», Boletín de la Real Sociedad Geográfica, t. CXIII (pp. 251-277).

- (1990): Clausewitz y su entorno intelectual, Madrid, Centro Superior de la Defensa, Monografías del CESEDEN.

Anuario militar de España (1936), Madrid, Imprenta y talleres del Ministerio de la Guerra.

ARANDA MATA, Antonio, General de Brigada de E. M. (1942): «Geografía cidiana», Boletín de la Real Sociedad Geográfica, t. LXXVIII, pp. 635-646. 
_ (1942 a): «Presente y porvenir de Marruecos», Boletín de la Real Sociedad Geográfica, t. LXXVIII, pp. 20-34.

AreilzA, José María, y CASTIELla, Fernando María (1941): Reivindicaciones de España, Madrid, Instituto de Estudios Políticos.

Bosque MAUReL, Joaquín (1992): Geografía y geógrafos en la España Contemporánea, Granada, Universidad de Granada.

Bosque MAUReL, Joaquín, et. al.(1984): «Geografía política, Geopolítica y Geografía militar en España (1940-1983)», II Coloquio Ibérico de Geografía, Barcelona, Sección de Geografía. Facultad de Geografía e Historia.

Bullón, Eloy (1931): Los estudios geográficos y el porvenir de España, Madrid, Publicaciones de la Real Sociedad Geográfica.

CARrero Blanco, Luis, Capitán de fragata de E. M. (1941): «La Geografía y la guerra naval», Boletín de la Real Sociedad Geográfica, t LXXVII, pp. 45-70.

- (1941 a): «La Geografía y la guerra naval», Boletín de la Real Sociedad Geográfica t. LXXVII, pp. 235-262.

(1942): «África y el mañana de Europa», África, n. ${ }^{\circ}$ 2, pp. 2-4.

- (1943): «El problema naval de España», Boletín de la Real Sociedad Geográfica, t. LXXIX, pp. 87-102.

- (1945): «Síntesis del desarrollo de la guerra en África», Boletín de la Real Sociedad Geográfica, t. LXXXI, pp. 484-502.

Cuerpos y servicios de Estado Mayor y auxiliares de Estado Mayor, (1940-1945), Madrid, Servicio geográfico del Ejercito

Díaz Villegas, José, Teniente coronel de E. M. (1940): Geografía militar de España. Países y mares limítrofes, Madrid, Servicio Geográfico y Cartográfico del Ejercito.

_ (1942): «Lecciones geopolíticas de la guerra actual», Boletín de la Real Sociedad Geográfica, t. LXXVIII, pp. 203-233.

(1942 a): «Nuestra tesis sobre Marruecos», África, n. ${ }^{\circ}$ 8, pp. 6-7.

— (1942 b): «África española. Un imperio que no lo es y lo será», África, n. ${ }^{\circ}$ 7, pp. 9-12.

- (1944): «España en África», Boletín de la Real Sociedad Geográfica, t. LXXIX, pp. 471-505.

— (1946): La Geografía y la guerra. Estudio militar del terreno, Madrid, Imprenta del Servicio Geográfico del Ejercito.

Dicenta Seguí, José, Capitán médico (1945): «Impresiones de la expedición de tropas españolas a nuestros territorios del Golfo de Guinea», África, n. ${ }^{\circ} 37-38$, pp. 24-28.

- (1945 a): «Parasitismo intestinal en el Golfo de Guinea» África, n. ${ }^{\circ}$ 41, pp. 24-27.

Domechen LAfuente, Ángel, Teniente coronel (1944): «La pesca en los Ait Ba», África, n. ${ }^{\circ} 35-36$, pp. 65-75.

- (1945): «La agricultura en Ifni. Agua para los Ait Ba Amram», África n. ${ }^{\circ} 41$, pp. 5-13.

Escalafoncillos de los cuerpos patentados de la armada (1940-1945), Madrid, Ministerio de la Marina.

Escalilla de las armas y cuerpos del ejercito del aire (1940-1945), Madrid, Ministerio del Aire.

Fontán y Lobé, Juan, Capitán de fragata de E. M. (1942): «El reparto de África», África, n. ${ }^{\circ}$, pp. $3-5$.

- (1942 a): «Poblaciones negras del África ecuatorial», África, n. ${ }^{\circ}$ 11, pp. 2-5.

GARcía FIgUeras, Tomás (1942): «Reivindicaciones de España en el Norte de África, África, n. ${ }^{\circ} 11$, p. 13.

GAZAPO VALCÁRCEL, Darío (1941): «La cartografía militar», Boletín de la Real Sociedad Geográfica, t. LXXVII, pp. 31-44.

Gómez DE ARTECHE y MoRo, José (1859): Geografía histórico-militar de España y Portugal, Madrid, Tipografía Francisco de P. Mellado. 
Gómez Mendoza, Josefina (1997): «La formación de la Escuela Española de geografía (1940-1952). Instituciones, revistas, congresos y programas», Eria, n. ${ }^{\circ} 42$, pp. 107146.

LACOSTE, Yves (1977): La geografía: un arma para la guerra, Barcelona, Anagrama.

LiNARES MAZA, Antonio, Capitán médico (1943): «El tipo humano en nuestro territorio del Ifni», África, n. ${ }^{\circ} 18$, pp. 35-37.

LOMBARDERo, Vicente Manuel, Coronel de E. M., Coronel de E. M. (1944): «Cartografía del Sahara español» África, n. ${ }^{\circ} 30$, pp. $28-37$.

- (1945): «Cartografía del África española», Boletín de la Real Sociedad Geográfíca, t. LXXXI, pp. 403-483.

LOSADA MALVÁREZ, Juan Carlos (1990): Ideología del ejército franquista, Madrid, Istmo.

Melón y Ruiz DE GoRdeJuela, Amando (1940): «Geopolítica o Geografía política su posible contenido", Estudios Geográficos, n. ${ }^{\circ}$ 2, pp. 5-33

MoREno LóPEZ, Eduardo (1912): Geografía general y de Europa, Barcelona, Tip. El Anuario de la exportación.

MostaZA, Bartolomé (1942): «Marruecos y nuestro espacio vital», África, n. ${ }^{\circ}$, pp. 31-34. (1942 a): «Mauritania y nuestra plenitud nacional», África, n. ${ }^{\circ}$ 7, pp. 46-49.

MunaIZ DE BREA, Ricardo, Teniente coronel del ejercito del aire, E. M. (1942): «El aire africano y su interés estratégico», África, n. ${ }^{\circ} 11, \mathrm{pp} .20-22$

— (1942 a): «La penetración aérea del continente negro» África, n. ${ }^{\circ}$ 6, pp. 16-19.

- (1943): «El aire español en la coyuntura presente y en el espacio estratégico euroafricano» África, n. ${ }^{\circ} 17$, pp. 20-21.

MUÑOZ I LLORET, Josep (1997): Jaume Vicens Vives. Una biografía intel-lectual, Barcelona, Edicions 62

Muro Morales, José Ignacio (1992): El pensamiento militar sobre el territorio en la España Contemporánea, Madrid, Ministerio de Defensa.

NÚÑEZ IGLESIAS, Pedro, Capitán de corbeta (1942): «El factor meteorológico en la paz y en la guerra», Boletín de la Real Sociedad Geográfica, t. LXXVIII, pp. 336-351.

Reguera Rodríguez, Antonio (1991): Fascismo y geopolítica en España, Barcelona, Geocrítica, n. ${ }^{\circ} 94$.

RoDRíGUEz EsTEBAN, José Antonio (1996): Geografía y colonialismo. La Sociedad Geográfica de Madrid (1876-1936), Madrid, Ediciones de la Universidad Autónoma.

RotaECHe, Jesús María, Capitán de navío (1942): «Pasado, presente y futuro de la Marina mercante», Boletín de la Real Sociedad Geográfica, t. LXXVIII, pp. 612-652.

Torroja, José María (1943): «Acta de sesiones», Boletín de la Real Sociedad Geográfica, t. XXXIX

Vicens VIves, J. (1940): Geopolítica del Imperio y del Estado, Barcelona, Editorial Yunque. (1950): Tratado general de geopolítica . Barcelona, Vicens Vives.

VILLANUEVA LÓPEZ-MORENo, Luis (1927): Bases para el estudio de la Geografía militar, Madrid.

RESUMEN: La geografía militar española (1939-1945). La geografía militar española durante el período 1939-1945 se caracterizó por seguir la tradición geográfica anterior a la guerra civil española, en la que se realizaban estudios estratégicos y tácticos al margen de las teoría geopolíticas que a partir de finales del siglo XIX predominaban en Europa. No obstante, debido a la influencia política de las potencias del Eje se dio una influencia, de la geopolítica alemana en un sector de la cúpula militar, siendo abandonada al finalizar la contienda mundial. En cambio, en los círculos del Derecho Internacional y perídisticos la penetración fue muy intensa, contrastándose con la geografía universitaria que mantuvo su rigor científico. 
PALABRAS CLAVE: Geografía militar, estrategia militar, táctica militar, geopolítica, espacio vital.

ABSTRACT: The military Spanish geography (1939-1945). The military Spanish geography during the period 1939-1945 was characterized by continuing the geographical tradition previous to the civil Spanish war, in which they were carried out strategic studies and tacticians to the margin of geopolitical theories that starting from ends of the XIX century prevailed in Europe. Nevertheless, due to the political influence of the powers of the Axis occurred an influence, of the German geopolitics in a sector of the military dome, being abandoned upon concluding the world war. On the other hand, in the circles of the International Right and journalistic the penetration was very intense, contrasting it with the university geography that maintained scientific rigor.

KEY WORDS: Military geography, military strategy, military tactics, geopolitics, vital space.

\section{ANEXO}

Relación de artículos publicados desde 1940 a 1945 en las revistas África y Boletín de la Real Sociedad Geográfica ordenados temáticamente.

\section{ARTÍCULOS DE GEOGRAFÍA GENERAL, LOCAL Y REGIONAL}

\section{Historia de las exploraciones geográficas}

Bullón, Galo (1945), Comandante, «Los Ulad Bu Sab del Sahara», África, n. ${ }^{\circ} 37-38$, pp. 4044.

Domenech la Fuente, Ángel, Teniente coronel (1945), «Algo sobre la colonia de Río de Oro», África, n. ${ }^{\circ} 44-45$, pp. $26-31$.

FonTÁN y LoBÉ, Juan, Capitán de fragata de E.M. (1942), «Temas de Guinea», África, n. ${ }^{\circ} 3$, pp. 3-6.

_ Capitán de fragata de E.M. (1943), «Apuntes para la Historia de África ecuatorial española. La expedición Argelejos», África, n. ${ }^{\circ}$ 18, pp. 3-5.

_ Capitán de fragata de E. M. (1943), «Apuntes para la Historia de nuestros territorios de Guinea», África, n. ${ }^{\circ}$ 17, pp. 2-4.

_ Capitán de fragata de E.M. (1943), «Las exploraciones españolas en la región occidental del África ecuatorial», África, n. ${ }^{\circ} 16$, pp. 3-6.

_ Capitán de fragata de E.M. (1943), «Las gestiones de venta y la expedición Lerena», África, n. ${ }^{\circ} 21$, pp. 3-6.

_- Capitán de fragata de E.M. (1943), «Notas para la historia de nuestras posesiones en Guinea. Consecuencia de la expedición Lerena. La expedición Manterola», África, n. ${ }^{\circ} 22$, pp. 3-6.

Galera Paniagua, Antonio, Coronel (1942), «Marruecos, algo sobre nuestra zona del protectorado», África, n. ${ }^{\circ} 3$, pp. 16-20.

LORENCI DE LA VEGA, Emilio, Comandante del ejercito del aire, E.M. (1945), «Cómo fue el desembarco y ocupación de Ifni», África, n. ${ }^{\circ} 39-40$, pp. 19-23

Medranos EzQuerre, Jesús María, Teniente coronel de E.M. (1942), «Santa Cruz de Mar Pequeña, África, n. ${ }^{\circ}$ 6, pp. 10-13. 


\section{Estudios locales}

Fontán y LoBÉ, Juan, Capitán de fragata de E.M. (1942), «Poblaciones negras del África ecuatorial», África, n. ${ }^{\circ} 11$, pp. 2-5.

LiNARES MAZA, Antonio, Capitán médico (1943), «El tipo humano en nuestro territorio del Ifni», África, n. ${ }^{\circ} 18$, pp. 35-37.

\section{Geografía de la salud}

DicENTA SEGUí, José, Capitán médico (1945», «Impresiones de la expedición de tropas españolas a nuestros territorios del Golfo de Guinea», África, n. ${ }^{\circ} 37-38$, pp. 24-28.

_-, José, Capitán médico (1945), «Parasitismo intestinal en el Golfo de Guinea», Áfri$c a$, n. $^{\circ} 41$, pp. $24-27$.

FonTÁN Y LOBÉ, Juan, Capitán de fragata de E.M. (1942), «El servicio sanitario colonial», África, n. ${ }^{\circ} 8$, pp. $2-5$.

\section{Geografía agraria}

Domenech la Fuente, Ángel, Teniente coronel (1944), «Arboles de los Ait Ba Amram», África, n. ${ }^{\circ} 28$, pp. $4-5$.

— Ángel, Teniente coronel (1945), «Arboles de los Ait Ba Amram. La palmera», África, n. ${ }^{\circ} 39-40$, pp. $4-8$.

—, Ángel, Teniente coronel, (1945), «La agricultura en Ifni. ¡Agua para los Ait Ba Amram!», África n. ${ }^{\circ} 41$, pp. 5-13.

FonTÁN y LoBÉ, Juan, Capitán de fragata de E.M. (1943), «Las producciones de la Guinea española. El cacao», África, n. ${ }^{\circ} 15$, pp. $2-5$.

MARTOREL OTZET, Vicente, Teniente coronel de ingenieros (1945), «Tetuán aplaca su sed», África, n. ${ }^{\circ} 37-38$, pp. 6-7.

\section{Geografía de las actividades marinas y pesqueras}

Domenech la Fuente, Ángel, Teniente coronel (1944) «La pesca en los Ait Ba», África, n. ${ }^{\circ}$ 35-36, pp. 65-75.

Rotaeche, Jesús María, Capitán de navío (1942), «Pasado, presente y futuro de la Marina mercante», Boletín de la Real Sociedad Geográfica, t. LXXVIII, pp. 612-652.

\section{Geografía local y regional}

Bullón DíAz, Galo (1945), Comandante, «Notas sobre Geografía humana de los territorios de Ifni y Sahara», Boletín de la Real Sociedad Geográfica, t. LXXXI, pp. 230-281.

DÍAZ DE VILEGAS, José (Hispanus), Teniente coronel de E.M., (1942), «Madagascar, posición estratégica», África, n. ${ }^{\circ}$ 5, pp. 24-27.

DomenECH LA FuENTE, Ángel, Teniente coronel, (1944) «El territorio de Ifni», África, n. ${ }^{\circ} 31-$ 32 pp. $55-62$.

Flores Morales, Ángel, Teniente topógrafo (1944), «La Granada Africana. Tlemgen, África, n. ${ }^{\circ} 33-34$, pp. 22-27.

— , teniente topógrafo (1945), «Geografía física, política y económica de Madagascar», Boletín de la Real Sociedad Geográfica, t. LXXXI, pp. 201-220.

_ Real Sociedad Geográfica, t. LXXX, pp. 740-757.

Gregori Peiró, Gonzalo, Teniente coronel (1943), «Tetuán la Blanca I.», África, n. ${ }^{\circ}$ 21, pp. 7-10.

—, Teniente coronel (1944), «Tetuán, la Blanca II.», África, n. ${ }^{\circ}$ 27, pp. 6-10.

Jiménez MendozA, Manuel, Interventor (1942), «Ciudades marroquíes del pasado. Bades», África, n. ${ }^{\circ} 12$, pp. $30-32$. 
LÓPEZ-CASTRO, Celestino, Teniente coronel (1944), «Rincones sevillanos y estampas marroquíes», África, n. ${ }^{\circ}$ 27, pp. 26-32.

- Teniente coronel (1945), «Torres y alminares. La Giralda fue «es-summa» de la gran aljama hispalense», Africa, n. ${ }^{\circ} 39-40$, pp. 15-18.

\section{Geografía histórica}

Aranda Mata, Antonio, General de Brigada de E.M. (1942), Geografía cidiana», Boletín de la Real Sociedad Geográfica, t. LXXVIII, pp. 635-646.

ARMADA SABAU, Ramón, Teniente coronel de E.M. (1945), «El faro de Alejandría», África, n. ${ }^{\circ} 37-38$, pp. 3-5.

Gallego Velasco, Miguel, Ingeniero militar de E.M. (1945), «El castillo de San Juan de Úlua, de Veracruz (Méjico), Boletín de la Real Sociedad Geográfica, t. LXXXI, pp. 681714.

GuILLÉN Y TATO, Julio, Capitán de fragata (1944), «Reseña histórica de los puertos de la baja Andalucía», Boletín de la Real Sociedad Geográfica, t. LXXX, pp. 309-311.

LÓPEZ-CASTRO, Celestino, Teniente coronel. (1945), «Añoranzas de la Toledo musulmana», África, n. ${ }^{\circ} 48$, pp. $14-17$.

\section{Artículos de aplicación militar}

\section{Cartografía}

GAZAPO VALDÉs, Darío, Coronel de E.M. (1941, «La cartografía militar», Boletín de la Real Sociedad Geográfica, t. LXXVII, pp. 31-44.

LOMBARDERo, Manuel, Coronel de E.M. (1944), «Cartografía del Sahara español», África, n. ${ }^{\circ} 30$, pp. $28-37$.

LOMBARDERO, Vicente Manuel, Coronel de E.M. (1945); «Cartografía del África española», Boletín de la Real Sociedad Geográfica, t. LXXXI, pp. 403-483.

\section{Meteorología}

CARRERo BlANCO, Luis, Capitán de fragata de E.M. (1941), «La Geografía y la guerra naval», Boletín de la Real Sociedad Geográfica, t. LXXVII, pp. 45-70.

NúNEEZ IGLESIAS, Pedro, Capitán de corbeta (1942), «El factor meteorológico en la paz y en la guerra, Boletín de la Real Sociedad Geográfica, t. LXXVIII, pp. 336-351.

Economía de guerra

González, Felix, Ingeniero militar (1941), «La economía de guerra», Boletín de la Real Sociedad Geográfica, t. LXXVII, pp. 217-234.

\section{Estrategia aérea}

MunAIZ DE BREA, Ricardo, Teniente coronel del ejercito del aire, E.M. (1942), «El aire africano y su interés estratégico», África, n. ${ }^{\circ} 11$, pp. $20-22$.

_ - Teniente coronel del ejercito del aire, E.M. (1942), «La penetración aérea del continente negro», África, n. ${ }^{\circ}$ 6, pp. 16-19.

_- Teniente coronel del ejercito del aire, E.M. (1943), «El aire español en la coyuntura presente y en el espacio estratégico euroafricano», África, n. ${ }^{\circ} 17$, pp. 20-21.

Evaluación militar de un territorio

Paschasius, Capitán (1942), «La marcha en el desierto», África, n. ${ }^{\circ}$ 2, pp. 31-36. 
Operaciones militares

Angosto, José, Teniente coronel de E.M. (1943) «La guerra en Libia y Egipto», África, n. ${ }^{\circ} 14$, pp. $32-34$.

CARRERo Blanco, Luis, Capitán de fragata de E.M (1945), «Síntesis del desarrollo de la guerra en África», Boletín de la Real Sociedad Geográfica, t. LXXXI, pp. 484-502.

DíAZ DE VILLEGAS, José (Hispanus), Teniente coronel de E.M. (1942), «La batalla líbica y la del canal de Sicilia», África, n. ${ }^{\circ} 2$, pp. 21-23.

García Figueras, Vicente, Teniente coronel de E.M. (1943), «El salto de África a Europa», África, n. ${ }^{\circ} 21$, pp. 18-23.

—, Teniente coronel de E.M. (1943), «El salto de África a Europa», África, n. ${ }^{\circ} 22$, pp. 27-30.

—, Teniente coronel de E.M. (1943), «El salto de África a Europa», África, n. ${ }^{\circ} 23$, pp. 36-41.

—, Teniente coronel de E.M. (1943), «El salto de África a Europa», África, n. ${ }^{\circ} 24$, pp. 29-32.

_ , Teniente coronel de E.M. (1944), «El salto de África a Europa», África, n. ${ }^{\circ} 25$, pp. 32-36.

_ , Teniente coronel de E.M. (1944), «El salto de África a Europa», África, n. ${ }^{\circ} 26$, pp. 31-36.

_ - Teniente coronel de E.M. (1944), «El salto de África a Europa», África, n. ${ }^{\circ} 27$, pp. 36-39.

—, Teniente coronel de E.M. (1944), «El salto de África a Europa», África, n. ${ }^{\circ} 28$, pp. 40-42.

_ , Teniente coronel de E.M. (1944), «El salto de África a Europa», África, n. ${ }^{\circ} 29$, pp. 33-37.

_ - Teniente coronel de E.M. (1944), «El salto de África a Europa», África, n. ${ }^{\circ} 30$, pp. 38-44.

—, Teniente coronel de E.M. (1944), «El salto de África a Europa», África, n. ${ }^{\circ} 31-32$, pp. 68-74.

—, Teniente coronel de E.M. (1944), «El salto de África a Europa», África, n. ${ }^{\circ} 33-34$, pp. 49-57.

_ , Teniente coronel de E.M. (1944), «El salto de África a Europa», África, n. ${ }^{\circ} 35-36$, pp. 58-64.

LÓPEZ-CASTRO, Celestino, Teniente coronel (1944), «Mi marcha y entrada en Tazarut», Áfri$c a$, n. ${ }^{\circ} 30$, pp. $14-18$.

MATEo Marcos, Santiago, Comandante de E.M. (1942), «El cuerpo del ejercito marroquí en la guerra de España», África, n. ${ }^{\circ}$ 9, pp. 4-8.

—, Teniente coronel de E.M. (1943), «La guerra en el norte de África durante el año 1942», África, n. ${ }^{\circ} 13$, pp. 6-10.

Munauz DE BREA, Ricardo, Teniente coronel del ejercito del aire, E.M. (1943), «El desembarco anglosajón en África», África, n. ${ }^{\circ}$ 14, pp. 17-20.

\section{Geopolítica naval}

CARRERo BLANCO, Luis, Capitán de fragata de E.M (1941), «La Geografía y la guerra naval», Boletín de la Real Sociedad Geográfica, t. LXXVII, pp. 235-262.

- Capitán de fragata de E.M. (1942), «África y el mañana de Europa», África, n. ${ }^{\circ}$ 2, pp. 2-4.

_ Capitán de fragata de E.M (1943), «El problema naval de España», Boletín de la Real Sociedad Geográfica, t. LXXIX, pp. 87-102.

DÍAZ DE VILLEGAS, José, Teniente coronel de E.M. (1942), «Lecciones geopolíticas de la guerra actual», Boletín de la Real Sociedad Geográfica, t. LXXVIII, pp. 203-233. 
GarCíA GarCíA, Juan, Teniente de navío (1942) «La navegación isocotémica», Boletín de la Real Sociedad Geográfica, t. LXXVIII, pp. 67-84.

RotaEche, Jesús María, Capitán de navío (1942), «La oceanografía en el Norte de África», África, n. ${ }^{\circ} 6$, pp. $2-5$.

Geopolítica (reivindicaciones territoriales)

ARANDa MATA, José, General de brigada de E.M. (1942), «Presente y provenir de Marruecos», África, n. ${ }^{\circ} 1$, pp. 3-9.

Aranda MATA, Antonio, General de Brigada de E.M. (1942), «Presente y porvenir de Marruecos», Boletín de la Real Sociedad Geográfica, t. LXXVIII, pp. 20-34.

DíAZ DE VILLEGAS, José (Hispanus), Teniente coronel de E.M. (1942), «África española. Un imperio que no lo es y lo será», África, n. ${ }^{\circ} 7$, pp. 9-12.

— Teniente coronel de E.M. (1942), «África, el continente de la injusticia. Cómo deshicieron el Imperio alemán y como medraron otros», África, n. ${ }^{\circ}$ 7. pp. 29-33

- (Hispanus), Teniente coronel de E.M. (01942), «Nuestra tesis sobre Marruecos», Áfri$c a$, n. $^{\circ} 8$, pp. 6-7.

- (Hispanus), Teniente coronel de E.M. (1944) «España en África», Boletín de la Real Sociedad Geográfica, t. LXXIX, pp. 471-505.

FonTÁN y LoBÉ, Juan, Capitán de fragata de E.M. (1942), «El reparto de África», África, n. ${ }^{\circ} 7$, pp. $3-4$. 